\title{
Shape of the neuroretinal rim and position of the central retinal vessels in glaucoma
}

\author{
Jost B Jonas, Martín C Fernández
}

\begin{abstract}
Glaucomatous neuroretinal rim loss can occur in a sequence of sectors with the temporal inferior disc sector as the first and the nasal superior disc sector as the last to be affected. This study evaluated whether the position of the central retinal vessel trunk is correlated with this pattern of glaucomatous rim loss. Morphometrically stereo colour optic disc photographs of 157 glaucomatous eyes and 67 normal eyes were checked. In the normal and glaucomatous eyes, the central retinal vessel trunk was located eccentrically in the upper nasal quadrant of the optic disc. Taking into account the vertically oval disc shape, the distance to the central vessel trunk was largest for the temporal inferior disc region and shortest for the nasal superior disc area. An abnormal form of the glaucomatous neuroretinal rim was found in eyes with an atypical location of the retinal vessel trunk. Also in these glaucomatous eyes, the rim loss was usually most and least marked in that sector with the longest and shortest distance, respectively, to the central retinal vessel trunk. One could infer that the sequence of rim loss in glaucoma is dependent upon the distance of the region to the central retinal vessel trunk; the further away the region from the retinal vessel trunk, the more likely it is to be affected by rim loss. This suggests that the distance from the central retinal vessels is one factor among others that is correlated with the regional vulnerability of the neuroretinal rim to the glaucomatous process. (Br f Ophthalmol 1994; 78: 99-102)
\end{abstract}

Glaucoma is associated with a diminution of optic nerve fibres leading to a decrease of neuroretinal rim area. ${ }^{12}$ This rim loss occurs in all sectors of the optic disc with regional preferences depending on the stage of the disease. In eyes with modest glaucomatous damage, rim loss is found predominantly at the inferotemporal and superotemporal disc regions..$^{3-6}$ In eyes with moderately advanced glaucomatous atrophy, the temporal horizontal disc region is the location with relatively the most marked rim loss. In very advanced glaucoma, the rim remnants are located mainly in the nasal disc sector, with a larger rim portion in the upper nasal region than in the lower nasal region. ${ }^{6}$ This sequence of disc sectors (inferotemporal - superotemporal temporal horizontal - nasal inferior - nasal superior) correlates with the progression of visual field defects with early perimetric changes in the nasal upper quadrant of the visual field and a last island of vision in the temporal inferior part of the visual field in eyes with almost absolute glaucoma. The present study was performed to evaluate whether the position of the central retinal vessel trunk is correlated with this pattern of glaucomatous neuroretinal rim loss.

\section{Materials and methods}

A total of 157 glaucomatous eyes (78 right eyes, 79 left eyes) of 87 glaucoma patients ( 35 men, 52 women; mean age: $65 \cdot 1$ (SD 10.9) years; range: 27-83 years, mean refractive error: $+0.02(2.06)$ dioptres; range: $-5 \cdot 6$ to $+6 \cdot 5$ dioptres), and 78 normal eyes (40 right eyes, 38 left eyes) of 48 subjects ( 27 men, 21 women, with a mean age of $51.0(17 \cdot 0)$ years; range: $8-78$ years; and a mean refractive error of $+0.39(1.44)$ dioptres; range: $-2 \cdot 25$ to $4 \cdot 0$ dioptres) were included in the study.

These were randomly selected eyes out of a set of normal and glaucomatous eyes in which the exit of the retinal vessel trunk on the lamina cribrosa surface was clearly visible. Eyes with a myopic refractive error exceeding -8 dioptres were excluded because of a different optic disc morphology.' Additionally, 53 glaucomatous eyes with tilted discs were evaluated. They were defined by a horizontally oval optic disc shape with the horizontal disc diameter being at least $10 \%$ longer than the vertical one. The ratio of the minimal disc diameter to the maximal disc diameter was at maximum $\mathbf{0 . 9 0}$.

Criteria for the diagnosis of glaucoma were an elevated intraocular pressure to readings of more than $21 \mathrm{~mm} \mathrm{Hg}$, glaucomatous changes of the optic nerve head such as an abnormally small neuroretinal rim area in relation to the optic disc size, larger vertical than horizontal cup to disc ratios, splinter-shaped optic disc haemorrhages, reduced visibility of the retinal nerve fibre bundles including localised defects, and glaucomatous visual field defects.

For all eyes, 15 degree colour stereo optic disc photographs had been taken using a telecentric Zeiss fundus camera equipped with an Allen stereo separator. The disc transparencies were projected in a scale of 1 to 15 . The outlines of the optic disc and optic cup were plotted on paper and morphometrically analysed. We measured area and horizontal, vertical, minimal, and maximal diameters of the disc and cup, and width of the neuroretinal rim every 30 degrees. The exit position of the central retinal artery and vein on the lamina cribrosa surface was measured as the distance between the centre of the vessel and the vertical optic disc axis and the horizontal optic disc axis. The optic cup was stereoscopically defined on the basis of contour and not of pallor. The border of the optic disc was identical to the inner side of the peripapillary scleral ring. The latter was a thin white band encircling the optic disc. To obtain values in absolute size units $(\mathrm{mm}$

Ophthalmology and Eye Hospital, University Germany Germany J B Jonas
} 
or $\mathrm{mm}^{2}$ ) the ocular and photographic magnification was corrected according to Littmann's method taking into account the ametropia and the anterior corneal curvature. ${ }^{8}$ The method has already been described in detail. ${ }^{2910}$ The photographs were evaluated in a masked fashion without knowledge of the clinical diagnosis and the visual field data.

For interindividual comparison, only one randomly selected eye per patient and subject was taken for statistical analysis. To examine the significance of differences, Wilcoxon and MannWhitney tests were applied.

\section{Results}

\section{POSITION OF THE CENTRAL RETINAL VESSEL}

\section{TRUNK}

In all normal and glaucomatous eyes, the retinal artery was located nasal to the retinal vein. In the normal eyes, the exits of the retinal artery and vein were located $0.21(0.13) \mathrm{mm}$ and 0.00 $(0 \cdot 12) \mathrm{mm}$, respectively, nasal to the vertical optic disc axis. For the glaucomatous eyes, similar values were obtained (artery: $0.24(0.20)$ $\mathrm{mm}$; vein: $0.05(0.19) \mathrm{mm}$ nasal to vertical disc axis) with no significant difference $(p=0.39$ (artery); $p=0.18$ (vein)) between the normal and glaucomatous eyes. Concerning the position in vertical direction, both the artery and vein exits were located on an average $0.02(0.11) \mathrm{mm}$ superior to the horizontal optic disc axis. The amount of the superior and nasal displacement of the central retinal vessel trunk was positively and significantly $(\mathrm{p}<0.01)$ correlated with the optic disc size. The larger the optic disc, the more pronounced was the displacement. The shape of the optic disc was slightly vertically oval with the vertical disc diameter $(1.89(0.25) \mathrm{mm})$ being about $6 \%$ longer than the horizontal one $(1 \cdot 78$ $(0 \cdot 19) \mathrm{mm})$. Taking into account this disc form and comparing the different disc sectors with each other, the temporal inferior disc border is the location with the longest distance to the central retinal vessel trunk. The nasal superior disc border shows the shortest distance to the central vessel trunk.

With respect to an abnormal position of the retinal vessels we compared glaucomatous eyes $(n=53)$ with the retinal vessel trunk located in the superior disc half and eyes with the retinal vessel exit positioned in the inferior disc half $(n=35)$. The neuroretinal rim at the superior disc margin was significantly $(p<0.05)$ wider and larger in the glaucomatous eyes with the retinal vessel trunk located in the superior disc region than in the eyes with the retinal vessel exit positioned in the inferior disc area (rim width at 12 o'clock: $0.22(0.12) \mathrm{mm}$ versus $0.17(0 \cdot 10)$ $\mathrm{mm}$; rim area in the temporal superior disc sector: $0.28(0.14) \mathrm{mm}^{2}$ versus $\left.0.22(0.12) \mathrm{mm}^{2}\right)$. Concerning an unusual location of the retinal vessel trunk in the horizontal direction, the neuroretinal rim in the temporal horizontal disc region was wider in the eyes with the retinal vessel trunk located in the temporal disc half $(n=22)$ than in the eyes with the retinal vessel exit positioned in the nasal disc half $(n=65)(0 \cdot 17$ $(0.08) \mathrm{mm}$ versus $0.14(0.11) \mathrm{mm})$. The rim in the nasal disc sector was wider in the glaucomatous eyes with the retinal vessel trunk in the nasal disc half $(n=65)$ than in the eyes with the retinal vessels exiting in the temporal disc half $(\mathrm{n}=22)(0.24 \quad(0.11) \mathrm{mm}$ versus $0.21 \quad(0.09)$ $\mathrm{mm})$. Total neuroretinal rim area did not differ significantly between the glaucoma subgroups with varying vessel location.

With respect to an abnormal optic disc shape, 53 glaucomatous eyes with tilted discs were evaluated. Due to the selection, they were characterised by a horizontally oval optic disc shape and an exit of the retinal vessels at the superior disc border. In these eyes, the neuroretinal rim was significantly $(p<0.01)$ smaller in the inferior disc region than in the superior one (width: 0.23 $(0.21) \mathrm{mm}$ versus $0.29(0.21) \mathrm{mm}$; rim area in the temporal inferior disc sector: $0.24(0.15)$ $\mathrm{mm}^{2}$ versus rim area in the temporal superior disc sector: $\left.0.29(0 \cdot 13) \mathrm{mm}^{2}\right)$. In the eyes with tilted discs and far advanced glaucoma, the rim remnants were significantly $(p<0.01)$ larger at the superior disc border than in the nasal, inferior, and temporal disc regions.

For the normal eyes, the configuration and width of the neuroretinal rim were statistically independent of the location of the retinal vessel exit. The optic disc size was not significantly different between the normal group $(2.73(0.59)$ $\left.\mathrm{mm}^{2}\right)$ and the glaucoma group $\left(2 \cdot 77(0 \cdot 78) \mathrm{mm}^{2}\right)$. These values are larger than those reported for unselected normal eyes $\left(2.69(0.70) \mathrm{mm}^{2}\right.$; not significant; Wilcoxon and Mann-Whitney test ${ }^{10}$ and glaucomatous eyes $\left(2.57(0.60) \mathrm{mm}^{2}\right.$; $\mathrm{p}<0.01) .{ }^{18}$

\section{Discussion}

Variables that may be associated with the pattern of glaucomatous rim loss ${ }^{3-6}$ are: (1) the physiological configuration of the rim being broader at the inferior and superior disc poles than at the nasal and temporal poles ${ }^{10}$; (2) the morphology of the inner surface of the lamina cribrosa with the larger pores and the higher ratio of pore to interpore connective tissue area in the inferior and superior regions compared with the temporal and nasal regions ${ }^{11-13}$; a high ratio of pore area to total area is considered to predispose to glaucomatous nerve fibre loss; (3) the glaucomatous bowing of the lamina cribrosa to the outside mainly in the inferior and superior disc regions as shown on scanning electron microscope photographs of glaucomatous eyes ${ }^{14}$; and (4) the regional distribution of thin and thick retinal nerve fibres with thin nerve fibres coming from the foveola, passing mainly through the temporal aspect of the optic disc and being less glaucoma susceptible than thick nerve fibres which originate predominantly in the fundus periphery, lead to the inferior, superior, and nasal disc regions ${ }^{15} 16$ and are more glaucoma sensitive than thin retinal ganglion cell axons. ${ }^{17}$ The latter parameter may explain why glaucomatous rim loss occurs later in the temporal horizontal disc sector with predominantly thin nerve fibres than in the temporal inferior or temperal superior disc sectors containing thin and thick axons. It is contradictory to the fact that in far advanced glaucoma rim remnants are usually located in the nasal disc sector. There, 
preferentially thick retinal ganglion cell axons leave the eye..$^{15}$

As an additional parameter for the pattern of glaucomatous rim loss, the distance from the central retinal vessel trunk in the lamina cribrosa may be considered. In both the normal and glaucomatous eyes of the present study, the retinal vessel trunk was slightly decentred into the nasal upper disc quadrant. Similar results have been obtained in a histomorphometric study of the lamina cribrosa surface. ${ }^{13}$ In the latter investigation, the exits of the retinal artery and vein were located $0.15(0.09) \mathrm{mm}$ and 0.07 $(0 \cdot 14) \mathrm{mm}$, respectively, nasal to the vertical optic disc axis and $0.02(0.11) \mathrm{mm}$ and 0.05 $(0.09) \mathrm{mm}$, respectively, superior to the horizontal optic disc axis. Taking into account the vertically oval optic disc shape, ${ }^{10}$ one may infer that the distance between the retinal vessels in the lamina cribrosa and the neuroretinal rim may be associated with the pattern of glaucomatous rim loss. The sector distant to the retinal vessel trunk is the temporal inferior disc region followed by the superior temporal and temporal horizontal disc regions. The nasal disc sector is the one closest to the retinal vessels with the nasal upper portion located closer to the retinal vessel trunk than the nasal inferior portion. In the same sequence, the glaucomatous rim loss has been reported to take place: temporal inferior temporal superior and temporal horizontal nasal inferior - nasal superior. ${ }^{3-6}$ Correspondingly, glaucomatous neuroretinal rim notches do not occur on an average at the inferior and superior disc poles but at about 13 degrees temporal to the vertical optic disc axis. ${ }^{18}$ These

Figure 1 Optic disc photographs of glaucomatous right eyes. Atypical positions of the central retinal vessel trunk correlated with an abnormal shape of the glaucomatous neuroretinal rim.

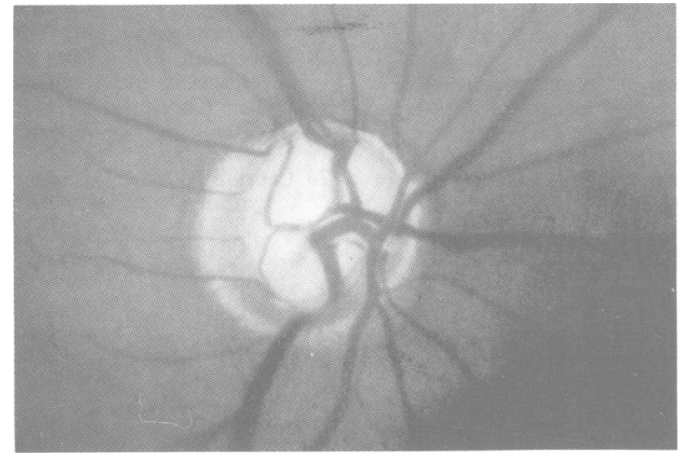

(A) Atypical rim notching in the nasal superior disc sector, retinal vessel trunk originating in the temporal inferior disc sector.

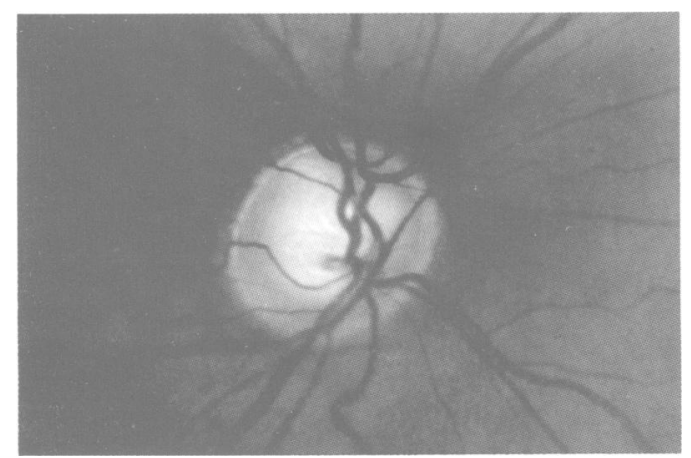

(B) Rim notching in the superior disc region, retinal vessel trunk located in the inferior disc region. Note: Corresponding to the rim loss, the parapapillary atrophy is larger in the superior region than in the inferior region. locations are more distant to the retinal vessel trunk than the vertical disc poles at the 6 o'clock and 12 o'clock position.

For strengthening the hypothesis that the local glaucoma susceptibility is correlated with the distance to the retinal vessel trunk, the following facts can be listed:

(1) In glaucomatous eyes with an unusual location of the retinal vessel trunk or an abnormal optic disc shape, the rim had an unusual form. Conversely, in eyes with an unusual glaucomatous rim configuration, the retinal vessel trunk was abnormally positioned (Figs 1A-C). Also in these glaucomatous eyes, the rim loss was usually most and least marked in that sector with the longest and shortest distance to the central retinal vessel trunk, respectively.

(2) The location of early glaucomatous visual field defects in the upper nasal or lower nasal field quadrants close to the horizontal line $\mathrm{e}^{19-22}$ indicates a ganglion cell loss in the region of the temporal fundus raphe. The axons of these ganglion cells lie in the deepest part of the retinal nerve fibre layer. ${ }^{2324}$ They are closest to the optic disc border. Within the optic disc, they are more distant to the central retinal vessel trunk than ganglion cell axons originating in the vicinity of the optic nerve head. These are located more centrally in the optic nerve head and are lost in a later stage of the disease.

Diagnostically, it may indicate that in eyes with an abnormal position of the central retinal vessels or an unusual optic disc form, early glaucomatous rim changes should be looked for not only in the temporal inferior disc sector but also in that disc sector that is most distant to the central retinal vessel trunk. It may explain why eyes with open angle glaucoma and a temporal cilioretinal artery retain longer central visual field than open angle glaucomatous eyes without a temporal cilioretinal artery. ${ }^{25}$

Pathogenetically, one may infer that the retinal vessel trunk could act as a stabilising element. It could render more difficult a mechanical distortion and backward bowing of the lamina cribrosa in glaucoma. This hypothesis is supported by photographs of a W-shaped lamina cribrosa in glaucomatous eyes. ${ }^{14}$ The lamina cribrosa is more condensed and bowed more to the back in the inferior and superior disc regions than close to the lamina cribrosa centre where the retinal vessels emerge. If the vessel trunk is decentred

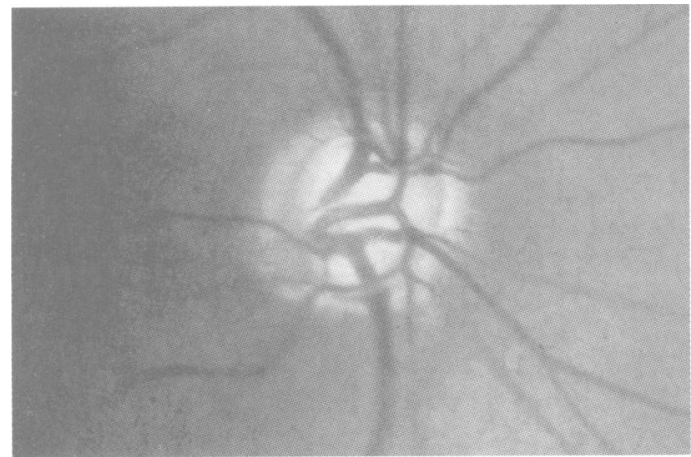

(C) Atypical position of the retinal vessel trunk in the inferior disc region correlating with rim remnants in the same disc area. Note: Corresponding to the rim loss, the parapapillary atrophy is larger in the superior region than in the inferior region. 
into the superior half of the optic disc, the inferior disc portion without vessel trunk support is larger than the superior one. Consequently, it can be deformed to a greater extent than the smaller superior disc part. It could explain the greater frequency of neuroretinal rim notches in the inferior temporal disc region compared with the superior temporal area. ${ }^{18}$

As a variation to this mechanical theory, one could also speculate that in the close vicinity of the retinal vessel trunk the vascular supply to the adjacent tissue is better than in the periphery. A vitally important participation of branches of the central retinal vessels in the nourishment of the optic nerve fibres in the lamina cribrosa, however, has not yet been demonstrated. ${ }^{26}$

As a factor limiting the scope of the present study one can mention that the position of the central retinal vessel trunk was measured in eyes in which the trunk on the lamina cribrosa surface was clearly detectable. This is possible only in discs showing central cupping. Due to the correlation between optic cup and optic disc size $^{102728}$ it implies that these selected optic discs were on average larger than normal optic nerve heads. In another histomorphometric investigation on the lamina cribrosa surface, however, the retinal vessel trunk was also located in the upper nasal quadrant. ${ }^{13}$ That study consisted of eyes of varying optic size including also small optic discs.

This study is part of a project of the Deutsche Forschungsgemeinschaft (Klinische Forschergruppe 'Glaukome', DFG ga 55/6-1/Jo).

1 Betz Ph, Camps F, Collignon-Brach J, Lavergne G, Weekers $R$. Biometric study of the disc cup in open-angle glaucoma. Graefes Arch Clin Exp Ophthalmol 1982; 218: 70-4.

2 Airaksinen PJ, Drance SM, Schulzer M. Neuroretinal rim area in early glaucoma. Am F Ophthalmol 1985; 99: 1-4.

in early glaucoma. Am 7 Ophthalmol $1985 ; 99: 1-4$.
Kirsch RE, Anderson DR. Clinical recognition of glauKirsch RE, Anderson DR. Clinical recognition of

4 Pederson JE, Anderson DR. The mode of progressive disc cupping in ocular hypertension and glaucoma. Arch Ophthalmol 1980; 98: 490-5.

5 Tuulonen A, Airaksinen PJ. Initial glaucomatous optic disk and retinal nerve fiber layer abnormalities and the mode of their progression. Am $\mathcal{f}$ Ophthalmol 1991; 111: 485-90.
6 Jonas JB, Fernández M, Stürmer J. Pattern of glaucomatous neuroretinal rim loss. Ophthalmology 1993; 99: 63-8.

7 Jonas JB, Gusek GC, Naumann GOH. Optic disc morphometry in high myopia. (A clinical study of 51 eyes.) Graefes metry in high myopia. (A clinical study of

8 Littmann H. Zur Bestimmung der wahren grösse eines Objektes auf dem Hintergrund des lebenden Auges. Klin Monatsbl Augenheilkd 1982; 180: 286-9.

9 Britton RJ, Drance SM, Schulzer MD, Douglas GR, Mawson DK. The area of the neuroretinal rim of the optic nerve in normal eyes. Am f Ophthalmol 1987; 103: 497-504.

10 Jonas JB, Gusek GC, Naumann GOH. Optic disc, cup, and neuroretinal rim size, configuration, and correlations in
normal eyes. Invest Ophthalmol Vis Sci 1988; 29: 1151-8.

11 Quigley HA, Addicks EM. Regional differences in the structure of the lamina cribrosa and their relation to glaucomatous optic nerve damage. Arch Ophthalmol 1981; 99: 137-43.

12 Radius RL. Regional specificity in anatomy at the lamina cribrosa. Arch Ophthalmol 1981; 99: 478-80.

13 Jonas JB, Mardin CY, Schlötzer-Schrehardt U, Naumann GOH. Histomorphometry of the human lamina cribrosa surface. Invest Ophthalmol Vis Sci 1991; 32: 401-5.

14 Quigley HA, Addicks EM, Green WR, Maumenee AE. Optic nerve damage in human glaucoma. II. The site of injury and

15 Sanchez RM, Dunkelberger GR, Quigley HA. The number and diameter distribution of axons in the monkey optic nerve. Invest Ophthalmol Vis Sci 1986; 27: 1342-50.

16 Jonas JB, Müller-Bergh JA, Schlötzer-Schrehardt UM Naumann GOH. Histomorphometry of the human optic nerve. Invest Ophthalmol Vis Sci 1990; 31: 736-44

17 Quigley HA, Sanchez RM, Dunkelberger GR, L'Hernault NL, Baginski TA. Chronic glaucoma selectively damages large optic nerve fibres. Invest Ophthalmol Vis Sci 1987; 28 large optic

18 Jonas JB, Gusek GC, Naumann GOH. Optic disc morphometry in chronic primary open-angle glaucoma. I. Morphometric intrapapillary characteristics. Graefes Arch Clin Ex Ophthalmol 1988; 226: 522-30.

19 Drance SM. The early field defects in glaucoma. Invest Ophthalmol 1969; 8: 84-91.

20 Hart WM Jr, Becker B. The onset and evolution of glaucomatous visual field defects. Ophthalmology 1982; 89: 268-79.

21 Gramer E, Gerlach R, Krieglstein GK, Leydhecker W. Topography of early visual field defects in computerized Topography of early visual field defects in computerized 22 Caprioli J, Sears M, Miller JM. Patterns of early visual field loss in open-angle glaucoma. Am $\mathcal{F}$ Ophthalmol 1987; 103: 512-7.

23 Radius RL, Anderson DR. The course of axons through the retina and the optic nerve head. Arch Ophthalmol 1979; 97 1154-8.

24 Minckler DS. The organisation of nerve fiber bundles in the primate optic nerve head. Arch Ophthalmol 1980; 98: $1630-6$.

25 Lee SS, Schwartz B. Role of the temporal cilioretinal artery in retaining central visual field in open-angle glaucoma. retaining central visual field

26 Liebran MF, Maumenee EA, Green RW. Histologic studies of the vasculature of the anterior optic nerve. studies of the vasculature of the
Am $₹$ Ophthalmol 1976; 82: $405-23$.

27 Bengtsson $B$. The variation and covariation of cup and disc diameters. Acta Ophthalmol 1976; 54: 804-18.

28 Caprioli J, Miller JM. Optic disc rim area is related to disc size in normal subjects. Arch Ophthalmol 1987, 105: 1683-5. 